\title{
Derivation of weighting coefficients for multiplexed phase-diffractive elements
}

\author{
E. Carcolé and M. S. Millán \\ Departament d'Òptica, Escola Universitària d'Òptica de Terrassa, Universitat Politècnica de Catalunya, \\ c/. Violinista Vellsolà No. 37, 08222 Terrassa, Spain \\ J. Campos \\ Department de Física, Universitat Autònoma de Barcelona, Edifici Cc, 08193 Barcelona, Spain
}

Received August 1, 1995

\begin{abstract}
We mathematically analyze the phase-only hologram constructed by taking the phase corresponding to the linear combination of two weighted phase functions. We show that this phase-only hologram may be written as a new linear combination for the original phase functions with new weights. Expressions for the new weights are developed to control the performance of the hologram. Also, some nondesired new terms appear and are written in terms of the original phase functions. The particular case of a bifocal lens is analyzed. (c) 1995 Optical Society of America
\end{abstract}

Diffractive optical elements are useful components in optical information processing systems. Array generators for optical interconnections, phase filters for correlation systems, beam shaping, adaptive optical components, and three-dimensional displays are common applications. Some of these applications require multiplexed functions, such as matched filters in pattern recognition and Fresnel lenses in optical interconnections. Suitable two-dimensional functions may be encoded in phase-modulating spatial light modulators for implementation of reconfigurable optical elements. Phase modulation has been shown with magneto-optic spatial light modulators, ${ }^{1}$ twistednematic liquid crystals, ${ }^{2}$ and transmissive matrix addressed ferroelectric liquid crystals. ${ }^{3}$ Research has been done on the optical interconnection capability of phase-only holograms corresponding to two multiplexed Fresnel lenses ${ }^{4,5}$ in which the output is a set of two different spots with different weights. Weighted multiplexed lenses forming different output images at different focal lengths ${ }^{6}$ or focusing in the same focal plane at different positions ${ }^{7}$ have also been considered. In Ref. 4 it was experimentally shown that the weights corresponding to hologram reconstruction have a nonlinear relation with the weights of the encoded linear combination, when the phase hologram corresponds to the linear combination of two weighted phase holograms. This problem forces one to use a variety of numerical techniques to compensate for the nonlinearities. $^{8-11}$ To our knowledge, no previous work mathematically deduced the nonlinear relation between the encoded weights and the resulting weights in the hologram reconstruction.

In this Letter we mathematically analyze the phase-only hologram constructed by taking the phase corresponding to the linear combination of two weighted phase functions. As a result, we show that a hologram constructed in this way may be written as a new linear combination of the original phase functions with new weights. Expressions for these weights are developed to control the performance of the hologram.
Some undesirable new terms appear and are written in terms of the original phase functions. Then the particular case in which the phase functions correspond to Fresnel lenses is considered, and the performance is analyzed. Finally, some conclusions are drawn.

We consider a linear combination of two phase functions $\exp (i \phi)$ and $\exp (i \theta) \quad(\phi$ and $\theta$ are twodimensional functions) with real weights $A$ and $B$ (we assume that $A^{2}+B^{2}=1$ throughout this Letter), which defines a new function $M \exp (i \alpha)$, where $M$ is the amplitude and $\alpha$ is the phase:

$$
M \exp (i \alpha) \equiv A \exp (i \phi)+B \exp (i \theta) .
$$

If we need $A$ and $B$ to be complex numbers, we may write $A=A^{\prime} \exp \left(i A^{\prime \prime}\right)$ and $B=B^{\prime} \exp \left(i B^{\prime \prime}\right)$, where $A^{\prime}$, $B^{\prime}, A^{\prime \prime}$, and $B^{\prime \prime}$ are real constants. Defining $\phi^{\prime}=$ $\phi+A^{\prime \prime}$ and $\theta^{\prime}=\theta+B^{\prime \prime}$, we may write the linear combination as $A^{\prime} \exp \left(i \phi^{\prime}\right)+B^{\prime} \exp \left(i \theta^{\prime}\right)$. This linear combination has the same properties as the one in Eq. (1). We need to perform some simple transformations in Eq. (1) to get a useful expression. By use of the Euler identities it is easy to verify the following equality:

$$
\begin{aligned}
M \exp (i \alpha)= & {\left[(A+B) \cos \left(\frac{\phi-\theta}{2}\right)+i(A-B)\right.} \\
& \left.\times \sin \left(\frac{\phi-\theta}{2}\right)\right] \exp \left(i \frac{\phi+\theta}{2}\right) .
\end{aligned}
$$

Writing the right-hand side of Eq. (2) in the polar representation we obtain for $M$

$$
M=\left[A^{2}+B^{2}+2 A B \cos (\phi-\theta)\right]^{1 / 2}
$$

and for $\exp (i \alpha)$

$$
\begin{aligned}
\exp (i \alpha)= & \exp \left(i \frac{\phi+\theta}{2}\right) \exp \left\{i \operatorname { a r c t a n } \left[\left(\frac{A-B}{A+B}\right)\right.\right. \\
& \left.\left.\times \tan \left(\frac{\phi-\theta}{2}\right)\right]\right\},
\end{aligned}
$$


where the $\arctan [. .$.$] function is assumed to give the$ phase of the complex number $(A+B) \cos [(\theta-\phi) / 2]+$ $i(A-B) \sin [(\theta-\phi) / 2]$ in Eq. (2); thus $\arctan [\ldots]$ gives values in the range $\{0,2 \pi\}$. Then $\exp (i \alpha)$ is the phase function to be encoded. The first part of Eq. (4) is the average value of the arguments of the original functions. The second part is a more complex function that requires a further transformation for its characteristics to be discovered. For simplicity, we define a new function and a new argument

$$
\begin{array}{r}
\exp [i \psi(\beta)] \equiv \exp \left\{i \arctan \left[\left(\frac{A-B}{A+B}\right) \tan (\beta)\right]\right\}, \\
\beta \equiv \frac{\phi-\theta}{2} .
\end{array}
$$

In Fig. $1 \psi(\beta)$ is plotted for different values of $a \equiv$ $A^{2}$. Note that the closer $A$ or $B$ is to zero, the more linear the resulting function. For $A \rightarrow B=0.5^{1 / 2}$ the limit function is a rectangle function. A key point for our development is that $\psi(\beta)$ can be considered a periodic function of $\beta$, with period $2 \pi$. This allows a Fourier series expansion to be performed:

$$
\exp [i \psi(\beta)]=\sum_{n} a_{n} \exp (\operatorname{in} \beta),
$$

where the weights $a_{n}$ can be written as

$$
a_{n}=\frac{1}{2 \pi} \int_{0}^{2 \pi} \exp \{i[\psi(\beta)-n \beta]\} \mathrm{d} \beta .
$$

The four main properties of the weights $a_{n}$ may be noted here: (i) the weights $a_{n}$ are real, because from Eq. (5) $\exp [i \psi(-\beta)]=\exp [-i \psi(\beta)]$. (ii) From Eq. (7) $a_{n}=0$ for $n$ even. (iii) From Eqs. (5) and (7) $a_{n}$ depends only on $(A-B) /(A+B)$ and $n$ and is completely independent of $\theta$ and $\phi$. (iv) For $B / A<0.7$, from Fig. 1 we may write $\psi(\beta)=\beta+\varepsilon(\beta)$, where $\varepsilon(\beta)$ is a function with period $\pi$ and a maximum value near $\pi / 4$. Then performing a first-order Taylor expansion, we may write $\exp [i \psi(\beta)] \approx(i \beta)[1+i \varepsilon(\beta)]$. Then the integrand in Eq. (7) becames $\exp [i(1-n) \beta]+$ $i \exp [i(1-n) \beta] \varepsilon(\beta)$. Note that $a_{1}$ takes a large value. For $n=-1,3$ the first part of the integrand does not contribute. To evaluate the second part, we may write $\varepsilon(\beta) \approx-\mu \sin (2 \beta)$, where $\mu$ is a small constant. Using this result we can write the integrand as $(-\mu / 2)\{\exp [i(3-n) \beta]-\exp [-i(1+n) \beta]\}$. This implies that $a_{3} / a_{-1} \approx-1$. For $B / A$ tending to 1 , $\psi(\beta)$ behaves as a step function, and the integral in Eq. (7) can be analytically evaluated and we get $a_{3} / a_{-1}=-1 / 3$. We conclude that the higher-order coefficients $(n>1, n<-1)$ are not negligible. Inserting Eq. (6) into Eq. (4) and using the definition of $\beta$ given in Eq. (5), we finally get

$$
\begin{aligned}
\exp (i \alpha)= & \sum_{n} a_{n} \exp \left\{i \frac{1}{2}[(1+n) \phi+(1-n) \theta]\right\} \\
= & a_{1} \exp (i \phi)+a_{-1} \exp (i \theta)+a_{3} \exp [i(2 \phi-\theta)] \\
& +a_{-3} \exp [i(2 \theta-\phi)]+\ldots
\end{aligned}
$$

This is the key result of this Letter. This means that the phase hologram corresponding to two multi- plexed phase functions may be written as a new linear combination of the original functions $(n=1$ and $n=-1$ ) affected by the new weights $a_{1}$ and $a_{-1}$. Undesired orders appear when other odd values of $n$ are considered.

To encode the multiplexed phase hologram, we need to know the relation between the input ratio $(A / B)^{2}$ and the desired ratio $\left(a_{1} / a_{-1}\right)^{2}$. This relation is plotted in Fig. 2(a) $\left[a_{1}\right.$ and $a_{-1}$ have been evaluated by Eq. (7) with numerical techniques ${ }^{12}$. The diffraction efficiency, defined as $a_{1}^{2}+a_{-1}^{2}$, is plotted as a func-

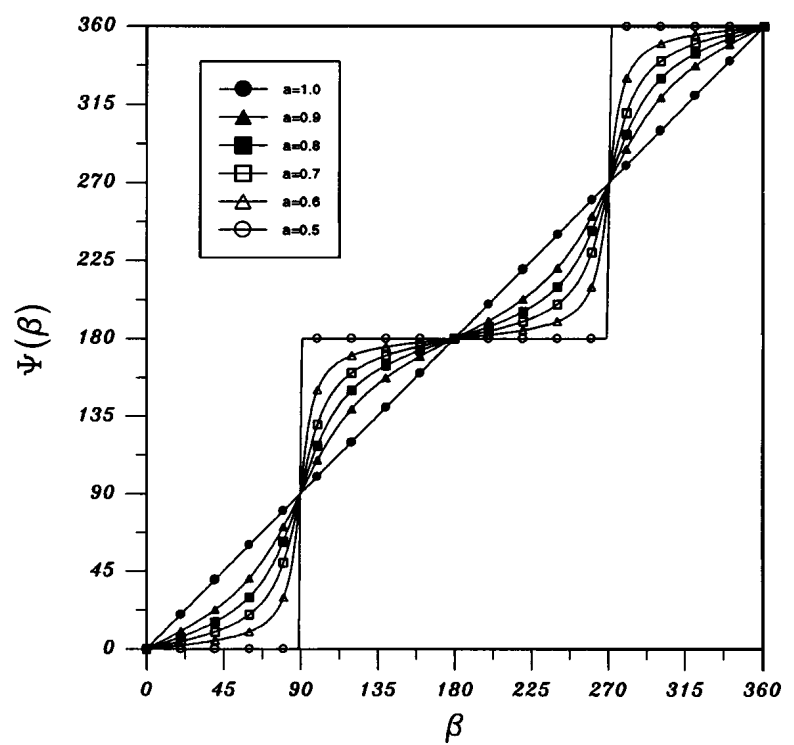

Fig. 1. $\psi(\beta)$ versus $\beta$ in degrees for different values of $a \equiv A^{2}$.

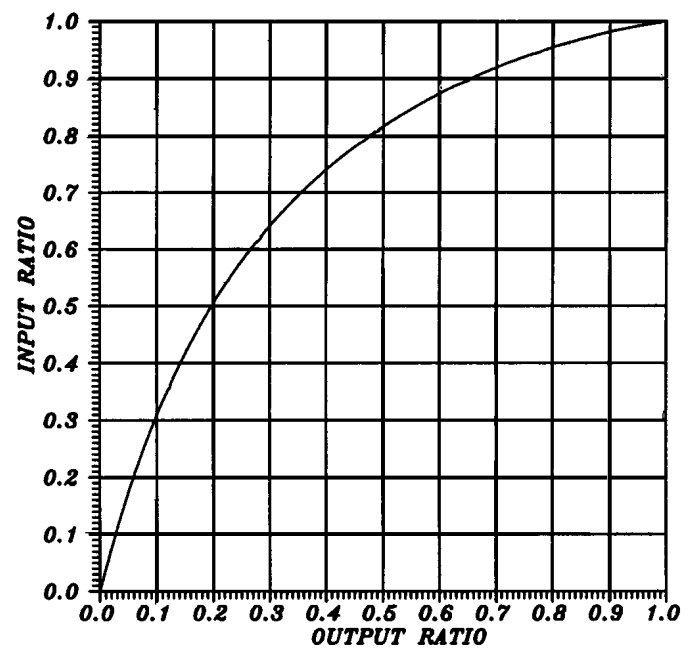

(a)

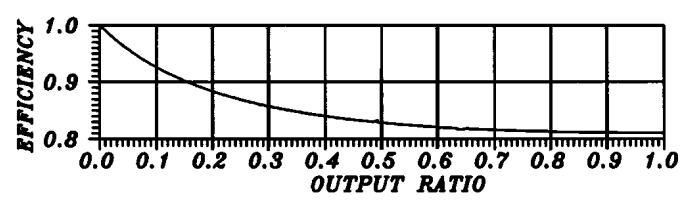

(b)

Fig. 2. (a) Input ratio and (b) diffraction efficiency versus the desired output ratio. 


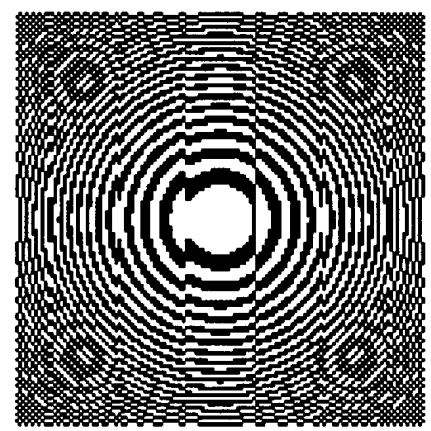

(a)

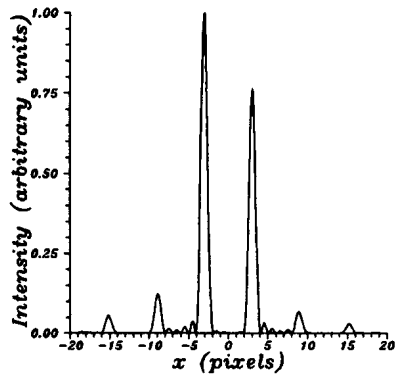

(b)
Fig. 3. Binary representation of a bifocal lens designed for $\left(a_{1} / a_{-1}\right)^{2}=0.75$ : (a) hologram, (b) reconstruction.

tion of the desired output ratio in Fig. 2(b). Note that the efficiency is always greater than 0.8 . To avoid numerical calculations for the hologram construction, one can perform an excellent sixth-degree polynomial fitting on the dependence of $(A / B)^{2}$ on $\left(a_{1} / a_{-1}\right)^{2}$ by using standard numerical techniques. ${ }^{12}$ When we define $x \equiv\left(a_{1} / a_{-1}\right)^{2}$ and $y \equiv(A / B)^{2}$ the polynomial is

$$
\begin{aligned}
y= & 0.000758498+3.90785 x-9.94724 x^{2} \\
& +19.3746 x^{3}-24.4142 x^{4} \\
& +16.9136 x^{5}-4.83813 x^{6} .
\end{aligned}
$$

The maximum absolute error of $y$ is less than 0.001 . This implies a fast and efficient way of obtaining the weights for the multiplexed hologram and thus possible real-time control of the weights.

The design of a bifocal lens is an example in which two phase functions are multiplexed. Many possibilities may be considered. We consider the particular case of two Fresnel lenses (quadratic phases) with the same focal length $f$, each shifted from the origin by an amount $\pm d / 2$ in the $x$ direction and with constant phase shifts of $\theta_{0}$ and $\phi_{0}$. In such a case $\theta=(k / 2 f)\left[(x-d / 2)^{2}+y^{2}\right]+\theta_{0}$ and $\phi=(k / 2 f)[(x+$ $\left.d / 2)^{2}+y^{2}\right]+\phi_{0}$, where $k$ is the wave number. Using this in Eq. (8), we may write the resulting bifocal lens as

$$
\begin{aligned}
\exp (i \alpha)= & \sum_{n} a_{n} \exp \left\{i \frac{k}{2 f}\left[\left(x+n \frac{d}{2}\right)^{2}+y^{2}\right]\right\} \\
& \times \exp \left\{i\left[\frac{k}{2 f}\left(1-n^{2}\right)\left(\frac{d}{2}\right)^{2}\right]\right. \\
& \left.+\frac{1}{2}\left(\phi_{0}+\theta_{0}\right)+\frac{1}{2} n\left(\phi_{0}-\theta_{0}\right)\right\} .
\end{aligned}
$$

Considering $n=1$ and $n=-1$, we obtain the desired shifted Fresnel lenses with the right phase shifts, $\theta_{0}$ and $\phi_{0}$. For other odd values of $n$, this expression predicts that other focalizations will appear at the positions $x=n d / 2$ ( $n$ odd) with an amplitude given by $a_{n}$. This completely agrees with the experimental results of Fig. 2(a) of Ref. 4. Note that the positions of the new focalizations do not depend on the original weights $A$ and $B$. A numerical example follows. If we wish that $x=\left(a_{1} / a_{-1}\right)^{2}=0.75$, from Eq. (9) we get $y=$ $(A / B)^{2}=0.937$. This implies that $A=0.695$ and $B=$ 0.718 . Using these values, we construct a bifocal lens [a binary representation is shown in Fig. 3(a)] with $128 \times 128$ square pixels whose size is $75 \mu \mathrm{m} \times 75 \mu \mathrm{m}$ and $d=450 \mu \mathrm{m}$ (6 pixels) with $f=1.138 \mathrm{~m}$ for a wavelength of $632.8 \mathrm{~nm}(\mathrm{He}-\mathrm{Ne}$ laser), as in Refs. 4-7. A section of the reconstruction of this hologram in the focal plane for plane-wave illumination is calculated by addition of the diffraction pattern of each square pixel. The result is shown in Fig. 3(b), which is in agreement with the expected ratio. Note also that the location of the peaks is $n d / 2$ with $d=6$ pixels, in agreement with Eq. (10).

In conclusion, the phase hologram constructed from the linear combination of two weighted phase functions has been studied mathematically. This study shows that the final hologram contains the linear combination of the original phase functions affected by new weights. Also, some undesirable additional terms arise. These new weights depend only on the original ones and not on the functions being multiplexed. A polynomial expression relating the desired final weights and the original ones has been developed to permit real-time control of the hologram performance. As a particular example, we studied the codification of multiplexed Fresnel lenses to discover their performance characteristics. It has been shown that some extra focalizations occur, and their locations have been determined. Finally, the method has a very high diffraction efficiency (in excess of 80\%).

We expect this work to be the first step in the development of mathematical expressions for more complex multiplexed holograms. This treatment is also useful for the multiplexing of two arbitrary phaseonly filters for pattern recognition purposes.

This research has been funded by projects TAP930667-C03-02 and TAP93-0667-C03-01. E. Carcolé thanks the Generalitat de Catalunya for a F.I. grant.

\section{References}

1. W. E. Ross, D. Psaltis, and R. H. Anderson, Opt. Eng. 22, 485 (1983).

2. J. C. Kirsch, D. A. Gregory, M. W. Thie, and B. K. Jones, Opt. Eng. 31, 963 (1992).

3. S. E. Broomfield, M. A. A. Neil, E. G. S. Paige, and G. G. Yang, Electron. Lett. 28, 26 (1992).

4. J. A. Davis and D. M. Cottrell, Opt. Lett. 19, 496 (1994).

5. J. A. Davis, R. P. Tiangco, T. H. Barnes, and T. G. Haskell, Appl. Opt. 34, 1929 (1995).

6. J. A. Davis, D. M. Cottrell, R. A. Lilly, and S. W. Connely, Opt. Lett. 14, 420 (1989).

7. J. A. Davis, S. W. Connely, G. W. Bach, R. A. Lilly, and D. M. Cottrell, Appl. Opt. 28, 102 (1989).

8. J. L. Horner and P. Gianino, Appl. Opt. 24, 851 (1985).

9. D. Casasent and W. A. Rozzy, Appl. Opt. 25, 3767 (1986).

10. R. R. Kallman, Appl. Opt. 25, 4216 (1986).

11. D. A. Jared and D. J. Ennis, Appl. Opt. 28, 232 (1989).

12. W. H. Press, B. P. Flannery, S. A. Teukolsky, and W. T. Vetterling, Numerical Recipes in C (Cambridge U. Press, Cambridge, 1990), pp. 111-141. 